$46^{\text {th }}$ Aerosciences Conference, Reno, Nevada

\title{
Gust Energy Extraction for Mini- and Micro- Uninhabited Aerial Vehicles
}

\author{
Jack W. Langelaan* \\ The Pennsylvania State University, University Park, PA 16802, USA \\ Götz Bramesfeld ${ }^{\dagger}$ \\ St. Louis University, St. Louis, MO 63103, USA
}

\begin{abstract}
This paper discusses energy extraction from atmospheric turbulence by small- and micro- uninhabited aerial vehicles. A nonlinear longitudinal dynamic model of a glider with elevators as the sole control input is used for the aircraft and feedback control laws for energy extraction are discussed. Using current measurements of wind speed and gradient the state which maximizes the gain in total energy is computed. A state feedback controller uses elevator input to regulate states to the optimal values. The state feedback control law is computed using LQR synthesis, and the state and input weight matrices which maximize energy gain are found using a search method. Simulation results of flights through sinusoidal gust fields and a thermal field show the performance of the proposed approach.
\end{abstract}

\section{Introduction}

A MAJOR HANDICAP associated with small- and micro- Unmanned Aerial Vehicles (smuAvs) is the limited on-board energy capacity (either as chemical fuel or as batteries). The reduced endurance and range which results greatly reduces the utility of such vehicles. Additionally, the low Reynolds numbers inherent to SMUAVs make it very difficult to achieve lift/drag ratios comparable to larger aircraft, further reducing overall performance.

However, significant energy is available from the atmosphere. Large birds and human sailplane pilots routinely exploit vertical air motion (lift) to remain aloft for several hours and fly hundreds of kilometers without flapping wings or the use of engines. Exploiting these long duration vertical air motions has been an active area of research for manned glider flight for many years and is now becoming of interest for small UAVs as well.

In addition to the relatively long-duration air motion exploited by sailplane pilots, energy is also available from short duration phenomena such as gusts. It has been observed by radio control glider pilots that flight performance relative to birds is significantly reduced on a gusty day. ${ }^{1}$ This implies that birds are exploiting gusts to minimize the effect on performance (and may in fact be able to improve performance), a feat which human RC pilots are not able to reproduce. Kiceniuk reports that it is even possible to extract energy from a downward gust ${ }^{2}$ ! Extracting energy from gusts is complicated by their typically short duration, hence very fast response (usually exceeding human reaction time) is required. While traditional flight control methods have focused on mitigating the effects of disturbances such as gusts, the research proposed here seeks to exploit disturbances to enhance range and endurance.

Urban environments are particularly gusty, and thus will greatly affect the flight performance of a small or micro air vehicle. Hence exploiting atmospheric disturbances such as gusts has the potential to significantly increase the utility of small flight vehicles operating in urban environments. While a significant amount of work has been done on exploiting longer-duration atmospheric effects (for example the autonomous soaring research described by Allen $^{3}$ ) and dynamic soaring (i.e. exploiting spatial gradients in a wind field ${ }^{1}$ ) less

*Assistant Professor, Department of Aerospace Engineering, Senior Member AIAA.

$\dagger$ Assistant Professor, Aerospace and Mechanical Engineering, Member AIAA

Copyright (C) 2008 by Jack W. Langelaan and Götz Bramesfeld. Published by the American Institute of Aeronautics and Astronautics, Inc. with permission. 
work has been performed on exploiting gusts. Phillips describes an approach to compute an equivalent thrust coefficient which occurs due to vertical gusts, ${ }^{4}$ and concludes that the effect is too small to be useful in crewed aircraft. However, extending Phillips approach to small UAVs shows that a significant performance improvement is possible.

One approach, which generated a control law based on lift coefficient, is described by Patel. ${ }^{5}$ An approach based on control of load factor is described by Lissaman. ${ }^{6}$ These early results show potential for energy savings of up to $40 \%$ when gust soaring is employed.

This paper presents a closed-loop architecture for gust energy extraction using a full model for aircraft longitudinal dynamics. While the methodology for control law development is general to powered SMUAVs and aircraft equipped with flaps, here we present results for a small autonomous glider with only elevator inputs.

The remainder of this paper is organized as follows: Section II provides a brief review of previous work; Section III discusses the dynamics and energetics of vehicle flight in gusts; Section IV describes the control architecture and control law development; Section V presents simulation results of flights through simusoidal gust fields and flight through a thermal field; and finally Section VI concludes the work.

\section{Previous and Related Research}

RIVEn BY COMPETITION glider flying, a significant amount of work has been reported on optimal piloting $D_{\text {techniques for static soaring. One of the most famous (and certainly most widely adopted) techniques }}$ was described by MacCready, ${ }^{7}$ which describes what is now known as MacCready speed to fly theory. Other selected examples include Arho, ${ }^{8}$ who examined minimum time soaring with a minimum altitude constraint, Metzger ${ }^{9}$ who described maximum speed with no net altitude loss, de Jong, ${ }^{10}$ who discussed a geometric approach to sailplane trajectory optimization, and somewhat more recently Cochrane, ${ }^{11}$ who extends MacCready theory to uncertain lift.

The trajectory optimization literature generally uses a simplified glider model, which assumes that the pilot has direct control of airspeed. This assumption is certainly appropriate for long duration flights where the glider spends most of its time in a trimmed condition, but this is assumption is not valid for periods of transition between trimmed conditions. Some authors have addressed optimal transitions to minimize energy loss, ${ }^{12,13}$ and elsewhere Gedeon ${ }^{14}$ describes an analysis of dolphin-style flight through thermals.

Dynamic soaring by both aircraft and birds has again become an active area of research. Optimal trajectories for energy extraction from wind gradients are described by Zhao ${ }^{15}$ and minimum fuel trajectories for power-assisted dynamic soaring are described by Zhao an Qi. ${ }^{16}$ Dynamic soaring using shear layers is described by Sachs, ${ }^{17}$ and elsewhere he discusses the minimum wind shear strength required for albatross flight. ${ }^{18}$ Pennycuick proposes an alternate flight mode where most of the energy gain is obtained from the shear layer which results from the winds flow separation over the crest of each wave. ${ }^{19}$ Successful exploitation of this strategy requires sensing very small changes in dynamic pressure, and he suggests that only tube-nosed birds such as albatrosses have the necessary sensory capability.

Both energy extraction from thermals and dynamic soaring are generally treated as deterministic problems. Gusts are inherently stochastic, are much shorter in duration, and generally show far greater spatial variation. This makes effective energy extraction more difficult. In addition, since useful energy extraction from gusts is only practical for small UAVs it has received comparatively less attention. Previously mentioned work by Lissaman, ${ }^{20}$ Patel $^{5}$ and Lissaman and Patel $^{6}$ uses a point mass model for the aircraft, thus ignoring potentially important dynamics.

\section{Vehicle Dynamics and Energetics}

$\mathrm{F}^{\mathrm{OR}}$ SIMPLICITY ONLY longitudinal motion is considered. Consider an aircraft located at $\mathbf{r}$ in an inertial $\Gamma$ frame $I$, where $\hat{x}^{i}$ and $\hat{z}^{i}$ define unit vectors (see Figure 1 ).

Using a common definition of stability axes, define $\hat{x}^{s}$ as a unit vector in the direction of airspeed (so that $\mathbf{v}=v_{a} \hat{x}^{s}$ ) and $\hat{z}^{s}$ opposite to lift. The velocity of the aircraft in the inertial frame is the sum of the velocity of the aircraft in the stability axes and the wind velocity:

$$
\dot{\mathbf{r}}=\mathbf{v}+\mathbf{w}
$$


Hence

$$
\ddot{\mathbf{r}}=\frac{\mathrm{d}}{\mathrm{d} t} \mathbf{v}+\frac{\mathrm{d}}{\mathrm{d} t} \mathbf{w}
$$

The angle $\gamma$ defines the rotation between the stability axes and the inertial axes, and it is the flight path angle with respect to the surrounding airmass. When $\mathbf{w}=0$ it is also the flight path angle with respect to the inertial frame. In this application $\gamma$ is defined as positive upwards, so for a steady glide the glideslope is negative. The acceleration of the aircraft is

$$
\frac{\mathrm{d}}{\mathrm{d} t} \mathbf{v}=\dot{v}_{a} \hat{x}^{s}+\boldsymbol{\omega}^{s} \times v_{a} \hat{x}^{s}
$$

Substituting $\boldsymbol{\omega}^{s}=\dot{\gamma} \hat{y}^{s}$ gives

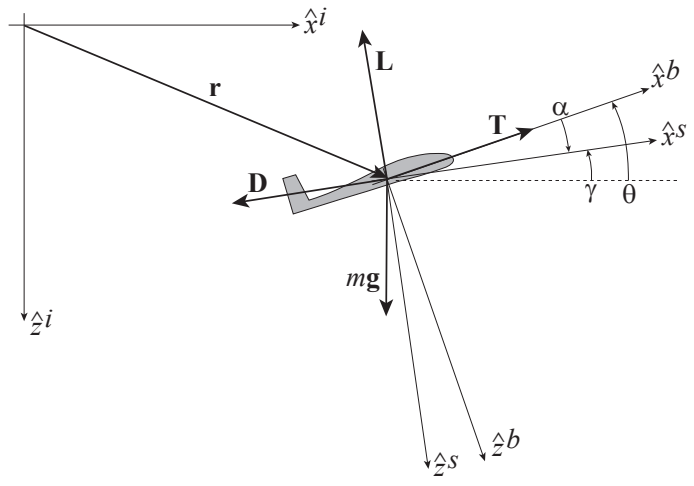

Figure 1. Reference frames. Positive rotations are indicated, so positive glideslope is upwards and angle of attack is positive in the conventional sense. $\frac{\mathrm{d}}{\mathrm{d} t} \mathbf{v}=\dot{v}_{a} \hat{x}^{\text {attack is }}-\dot{\gamma} v_{a} \hat{z}^{s}$

Therefore

$$
\mathbf{L}+\mathbf{D}+m \mathbf{g}+\mathbf{T}=m\left[\dot{v}_{a} \hat{x}^{s}-\dot{\gamma} v_{a} \hat{z}^{s}+\frac{\mathrm{d}}{\mathrm{d} t} \mathbf{w}\right]
$$

where $\mathbf{L}$ is the lift vector, $\mathbf{D}$ is the drag vector, $\mathbf{g}$ is the acceleration due to gravity, $\mathbf{T}$ is the thrust vector and $m$ is the mass. Lift and drag are generally expressed in the stability frame, thrust is generally expressed in the body frame and gravity is generally expressed in the inertial frame:

$$
\begin{aligned}
\mathbf{L} & =-\frac{1}{2} \rho v_{a}^{2} S C_{L} \hat{z}^{s} \\
\mathbf{D} & =-\frac{1}{2} \rho v_{a}^{2} S C_{D} \hat{x}^{s} \\
\mathbf{T} & =\frac{1}{2} \rho v_{a}^{2} S C_{T} \hat{x}^{b} \\
\mathbf{g} & =g \hat{z}^{i}
\end{aligned}
$$

The kinematics of the aircraft can now be defined in terms of the airspeed, flight path angle and wind speed. It is generally more convenient to work in terms of pitch angle and angle of attack, Figure 1 shows that $\gamma=\theta-\alpha$ :

$$
\begin{aligned}
\dot{x}_{i} & =v_{a} \cos (\theta-\alpha)+w_{x} \\
\dot{z}_{i} & =-v_{a} \sin (\theta-\alpha)+w_{z} \\
\dot{\theta} & =Q
\end{aligned}
$$

where $Q$ is pitch rate.

Vehicle dynamics are written in stability axes as

$$
\begin{aligned}
\dot{v}_{a} & =q \frac{S}{m}\left(C_{T} \cos \alpha-C_{D}\right)-\frac{\mathrm{d} w_{x}}{\mathrm{~d} t} \cos (\theta-\alpha)+\left(\frac{\mathrm{d} w_{z}}{\mathrm{~d} t}-g\right) \sin (\theta-\alpha) \\
\dot{\alpha} & =Q-q \frac{S}{v_{a} m}\left(C_{L}+C_{T} \sin \alpha\right)-\frac{1}{v_{a}} \frac{\mathrm{d} w_{x}}{\mathrm{~d} t} \sin (\theta-\alpha)-\frac{1}{v_{a}}\left(\frac{\mathrm{d} w_{z}}{\mathrm{~d} t}-g\right) \cos (\theta-\alpha) \\
\dot{Q} & =q \frac{S c C_{m}}{I_{y y}}
\end{aligned}
$$

where $q=\frac{1}{2} \rho v_{a}^{2}$.

In this research the wind field is assumed to be constant, hence

$$
\frac{\mathrm{d}}{\mathrm{d} t} \mathbf{w}=\frac{\mathrm{d}}{\mathrm{d} t}\left[\begin{array}{c}
w_{x} \\
w_{z}
\end{array}\right]=\nabla \mathbf{w}\left[\begin{array}{c}
\dot{x} \\
\dot{z}
\end{array}\right]=\left[\begin{array}{c}
\frac{\delta w_{x}}{\delta x_{i}} \dot{x}_{i}+\frac{\delta w_{x}}{\delta z_{i}} \dot{z}_{i} \\
\frac{\delta w_{z}}{\delta x_{i}} \dot{x}_{i}+\frac{\delta w_{z}}{\delta z_{i}} \dot{z}_{i}
\end{array}\right]
$$


The aerodynamic coefficients are

$$
\begin{aligned}
C_{L} & =C_{L 0}+C_{L_{\alpha}} \alpha+\frac{c}{2 v_{a}}\left(C_{L_{Q}} Q+C_{L_{\dot{\alpha}}} \dot{\alpha}\right)+C_{L_{\delta_{e}}} \delta_{e}+C_{L_{\delta_{f}}} \delta_{f} \\
C_{D} & =f_{L D}\left(C_{L 0}+C_{L_{\alpha}} \alpha\right)+C_{D_{\delta_{e}}} \delta_{e}+C_{D_{\delta_{f}}} \delta_{f} \\
C_{m} & =C_{m 0}+C_{m_{\alpha}} \alpha+\frac{c}{2 v_{a}} C_{m_{Q}} Q+C_{m_{\delta_{e}}} \delta_{e}+C_{m_{\delta_{f}}} \delta_{f}
\end{aligned}
$$

where $f_{L D}\left(C_{L 0}+C_{L_{\alpha}} \alpha\right)$ is a polynomial function which relates drag coefficient to lift coefficient. Control inputs are thrust coefficient $C_{T}$, elevator deflection $\delta_{e}$ and flap deflection $\delta_{f}$.

\section{A. Total Energy}

The vehicle's specific total energy (i.e. total energy divided by mass) is

$$
E_{t o t}=g h+\frac{1}{2}\left(\dot{x}_{i}^{2}+\dot{z}_{i}^{2}\right)
$$

where $h$ is height above a datum. Substituting vehicle kinematics,

$$
E_{t o t}=g h+\frac{1}{2}\left(v_{a}^{2}+2 v_{a} w_{x} \cos \gamma-2 v_{a} w_{z} \sin \gamma+w_{x}^{2}+w_{z}^{2}\right)
$$

The rate of change of specific energy is

$$
\begin{aligned}
\dot{E}_{t o t}= & g \dot{h}+v_{a} \dot{v}_{a} \\
& +\dot{v}_{a} w_{x} \cos \gamma+v_{a} \dot{w}_{x} \cos \gamma-\dot{\gamma} v_{a} w_{x} \sin \gamma \\
& -\dot{v}_{a} w_{z} \sin \gamma-v_{a} \dot{w}_{z} \sin \gamma-\dot{\gamma} v_{a} w_{z} \cos \gamma \\
& +\dot{w}_{x} w_{x}+\dot{w}_{z} w_{z}
\end{aligned}
$$

Gathering terms and letting $\dot{h}=v_{a} \sin \gamma-w_{z}$,

$$
\begin{aligned}
\dot{E}_{t o t}= & g\left(v_{a} \sin \gamma-w_{z}\right) \\
& +\dot{v}_{a}\left(v_{a}+w_{x} \cos \gamma-w_{z} \sin \gamma\right) \\
& -\dot{\gamma}\left(v_{a} w_{x} \sin \gamma+v_{a} w_{z} \cos \gamma\right) \\
& +\dot{w}_{x}\left(v_{a} \cos \gamma+w_{x}\right)+\dot{w}_{z}\left(-v_{a} \sin \gamma+w_{z}\right)
\end{aligned}
$$

Substituting vehicle dynamics and setting $\theta-\alpha=\gamma$,

$$
\begin{aligned}
\dot{E}_{t o t}= & q \frac{S}{m}\left[\left(v_{a} \cos \alpha+w_{x} \cos \theta-w_{z} \sin \theta\right) C_{T}\right. \\
& -\left(w_{x} \sin \gamma+w_{z} \cos \gamma\right) C_{L} \\
& \left.-\left(v_{a}+w_{x} \cos \gamma-w_{z} \sin \gamma\right) C_{D}\right] \\
& -v_{a}\left(\dot{w}_{x} \cos \gamma-\dot{w}_{z} \sin \gamma\right)-w_{x} \dot{w}_{x}-w_{z} \dot{w}_{z}
\end{aligned}
$$

And finally,

$$
\begin{aligned}
\dot{E}_{t o t}= & q \frac{S}{m}\left[\left(v_{a} \cos \alpha+w_{x} \cos \theta-w_{z} \sin \theta\right) C_{T}\right. \\
& -\left(w_{x} \sin \gamma+w_{z} \cos \gamma\right) C_{L} \\
& \left.-\left(v_{a}+w_{x} \cos \gamma-w_{z} \sin \gamma\right) C_{D}\right] \\
& -\mathbf{v}_{a}^{T}[\nabla \mathbf{w}] \mathbf{v}-2 \mathbf{v}_{a}^{T}[\nabla \mathbf{w}] \mathbf{w}-\mathbf{w}^{T}[\nabla \mathbf{w}] \mathbf{w}
\end{aligned}
$$

where $\mathbf{v}_{a}^{T}=\left[\begin{array}{ll}v_{a} \cos \gamma & -v_{a} \sin \gamma\end{array}\right]$ (i.e. airspeed expressed in the inertial frame) and $\nabla \mathbf{w}$ is the gradient of the wind vector, also expressed in the inertial frame.

The last three terms in Equation 25 define the contribution of wind gradient to power. Remembering that $z$ is positive down, we can see that negative gradients contribute to positive power (i.e. increasing upwards wind, increasing headwind allows power extraction from the gradient). 


\section{B. Trajectory Optimization}

Given the vehicle dynamics, the energy extraction problem can be cast as a trajectory optimization problem:

$$
\begin{array}{rc}
\operatorname{minimize} & C(\mathbf{x}, \mathbf{u}) \\
\text { subject to } & \dot{\mathbf{x}}=f(\mathbf{x}, \mathbf{u}, \mathbf{w}) \\
& \mathbf{x}_{\min } \leq \mathbf{x} \leq \mathbf{x}_{\max } \\
& \mathbf{u}_{\min } \leq \mathbf{u} \leq \mathbf{u}_{\max }
\end{array}
$$

where $C$ is a cost function (discussed below), Equation 27 defines constraints on vehicle dynamics, Equation 28 defines constraints on the state (for example minimum altitude or minimum/maximum airspeed), and finally Equation 29 defines constraints on control inputs (for example control surface deflections).

The choice of cost function is critical in the trajectory which is eventually computed, and may vary depending on mission. For example, maximum endurance can be obtained by minimizing power, maximum range can be obtained by maximizing $\mathrm{L} / \mathrm{D}$, minimum time can be obtained by maximizing velocity.

Here we will maximize $\frac{\Delta E}{\Delta x}$, the change in total energy with respect to distance. Note that in gliding flight in still air this quantity will always be negative, representing energy loss (a steady loss of altitude when airspeed is constant). For gliding flight:

$$
\begin{aligned}
\frac{\Delta E}{\Delta x}=\frac{\dot{E}}{\dot{x}}= & -\frac{1}{\left(v_{a} \cos \gamma+w_{x}\right)} q \frac{S}{m}\left[\left(w_{x} \sin \gamma+w_{z} \cos \gamma\right) C_{L}+\left(v_{a}+w_{x} \cos \gamma-w_{z} \sin \gamma\right) C_{D}\right] \\
& +v_{a}\left(\dot{w}_{x} \cos \gamma-\dot{w}_{z} \sin \gamma\right)+w_{x} \dot{w}_{x}+w_{z} \dot{w}_{z}
\end{aligned}
$$

In the dynamic case, simply computing $C_{L}$ to maximize instantaneous $\frac{\Delta E}{\Delta x}$ will result in minimizing $C_{L}$ (i.e. "pushing the nose down" to maximize airspeed). To obtain a useful solution one must compute a sequence of optimal $\left[\begin{array}{lll}v_{a} & C_{L} & \gamma\end{array}\right]$ over some finite time horizon (e.g. one period of a sinusoidal gust, as in Lissaman and Patel $^{6}$ ). However, this requires knowledge of future wind speeds, which in the case of stochastic gusts is unavailable.

A second approach (used here) is to compute the $C_{L}$ which maximizes energy gain (or equivalently, minimizes energy loss) while imposing steady glide constraints. In a steady glide,

$$
\begin{aligned}
L & =m g \cos \gamma=q S C_{L} \\
D & =-m g \sin \gamma=q S C_{D}
\end{aligned}
$$

hence $-\tan \gamma=\frac{C_{D}}{C_{L}}$. Further, assuming $\gamma$ is small,

$$
C_{L}=\frac{m g}{q S}
$$

and the instantaneous energy change for a particular value of $\mathbf{w}$ and $\nabla \mathbf{w}$ is now a function of only airspeed. ${ }^{\mathrm{a}}$

The optimal (steady state) airspeed can be found using standard bounded function minimization tools such as MatLab's fminbnd and the angle of attack can be computed from the associated optimal lift coefficient. The state which maximizes energy change is denoted $\mathbf{x}_{s s, o p t}$ and it comprises $\left[\theta_{s s, o p t} v_{a_{s s, o p t}} \alpha_{s s, o p t} 0\right]$. Note that the optimal pitch rate is zero in this steady state approximation.

Clearly the airspeed and angle of attack computed using this approach is suboptimal, since it does not allow full exploitation of the aircraft dynamics. However, the steady state computation allows us to determine an airspeed and angle of attack using only information available at the current instant: it does not require predictions of future wind, which are required by cyclic or receding horizon approaches.

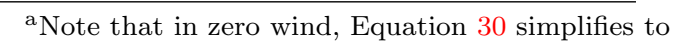

$$
\frac{\dot{E}}{\dot{x}}=-\frac{1}{v_{a} \cos \gamma} q \frac{S}{m} v_{a} C_{D}=-g \frac{C_{D}}{C_{L}}
$$

so maximizing energy change means minimizing $\frac{C_{D}}{C_{L}}$, i.e. flying at best L/D. 


\section{Control Architecture}

Now that an optimal vehicle state has been computed (or, in the case of a receding horizon control, a sequence of optimal states) a command following controller must be designed and implemented. A standard approach is is to design an inner loop controller (for example, capable of following commanded states or desired outputs) with the outer loop comprising the receding horizon control computation. This is shown schematically in Figure 2.

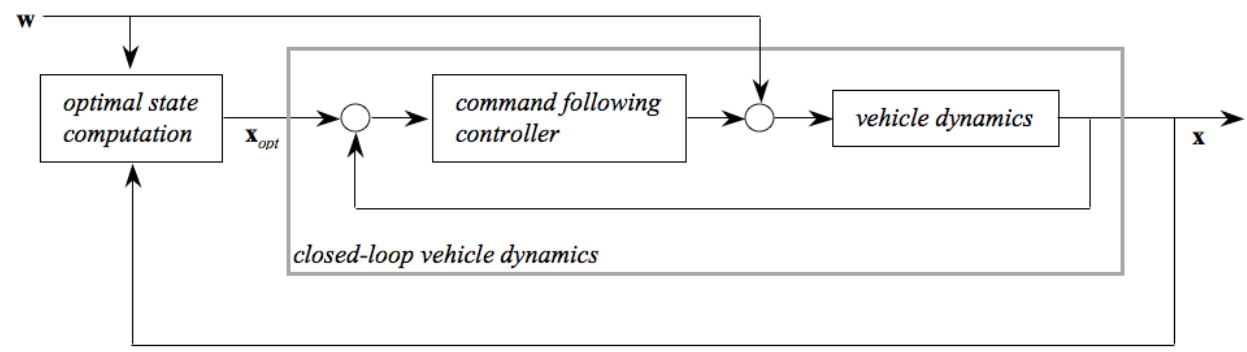

Figure 2. Inner/outer loop control employing energy maximization computation. This energy maximization may be a receding horizon controller (if some a priori knowledge of wind field is available).

As discussed above, in the research presented here the assumption of steady glide precludes the necessity for predictive control, and only the current wind is needed for computing the optimal state. In effect this is a receding horizon controller with a one step planning horizon.

The remainder of this paper is concerned with the inner loop control problem. Actually following commanded states in gusty conditions using limited control inputs (e.g. only elevators) is a difficult problem, and the ability to follow the commanded states will have a large effect on actual energy extraction. The problem, therefore, is designing an inner-loop controller that maximizes energy extraction given the commanded sequence of optimal states while respecting limitations such as saturation.

While the methodology presented here is general, as an example a closed loop controller is designed for an RnR Products SB-XC aircraft (parameters given in Table 2 of the Appendix). This aircraft is equipped with flaps, but here only elevator control is considered.

\section{A. Inner Loop Control Design}

To simplify the problem of feedback control, system dynamics can linearized about some trim condition (here, steady flight at best L/D airspeed) and a state feedback controller can be designed for small perturbations about this trim condition:

$$
\delta_{e}=-\mathbf{K}\left(\mathbf{x}-\mathbf{x}_{\text {trim }}\right)+\delta_{e, \text { trim }}
$$

where $\delta_{e}$ is elevator deflection.

In the implementation considered here the controller attempts to regulate the states to $\mathbf{x}_{s s, o p t}$, so

$$
\delta_{e}=-\mathbf{K}\left(\mathbf{x}-\mathbf{x}_{s s, o p t}\right)+\delta_{e, t r i m}
$$

The gain matrix $\mathbf{K}$ can be determined using techniques such as pole placement or LQR. This approach assumes that the change in linearized system dynamics is small over the range of states likely to be encountered during flight through the gust field, so a particular choice of $\mathbf{K}$ is appropriate over a wide speed range. This approach also assumes that $\delta_{e, t r i m}$ does not change significantly. Both these assumptions are likely to make the final solution brittle, but in the simulation results presented here good results are still obtained. It may be possible to use techniques such as gain scheduling to improve performance.

Here a controller was designed using a linear quadratic regulator, which seeks to minimize a cost function $J_{c}$ :

$$
J_{c}=\int\left(\left(\mathbf{x}-\mathbf{x}_{d e s}\right)^{T} \mathbf{Q}\left(\mathbf{x}-\mathbf{x}_{d e s}\right)+\mathbf{u}^{T} \mathbf{R} \mathbf{u}\right) d t
$$

where the state vector is $\mathbf{x}^{T}=\left[\begin{array}{llll}\theta & v_{a} & \alpha\end{array}\right]^{T}, \mathbf{x}_{\text {des }}$ is the desired state vector and the input is $\mathbf{u}=\delta_{e}$. The components of $\mathbf{Q}$ and $\mathbf{R}$ represent the relative importance of regulating a particular state and the control cost. 
The problem now is the choice of weight matrices $\mathbf{Q}$ and $\mathbf{R}$ which will maximize energy gain in the control architecture of Figure 2. For example, a large value of $Q_{33}$ and small value of $Q_{11}$ implies that angle of attack must be controlled more precisely than pitch angle.

Since $\mathbf{Q}$ and $\mathbf{R}$ define relative importance, we can set a particular component to 1 without loss of generality. To limit the dimension of the space of possible controllers, $Q_{33}$ was set to $1, Q_{44}$ was set to $1 \times 10^{-9}$ and $\mathbf{R}$ was set to 0.1 . The small value of $Q_{44}$ was chosen because the optimal value of pitch rate determined using the steady state approximation is zero, and the true optimal value of pitch rate is likely to be significantly different for the dynamic case. The value of 0.1 for $\mathbf{R}$ (which defines the cost of control input) was chosen after some experimentation. The remaining free parameters are $Q_{11}$ and $Q_{22}$, the relative importance of pitch angle and airspeed with respect to angle of attack, respectively.

Here we determine the optimal state cost and control cost to maximize energy change for flight through a sinusoidally varying vertical gust field. The gust field is assumed to be frozen in space, i.e.

$$
w_{z}\left(x_{i}\right)=\sqrt{2} \sigma_{z} \sin \frac{2 \pi x_{i}}{L_{w}}
$$

In this example the gust length $L_{w}=50 \mathrm{~m}$ and the root mean square vertical velocity $\sigma_{z}=2 \mathrm{~m} \mathrm{~s}^{-1}$. Simulations of flight $1000 \mathrm{~m}$ in length through the gust field using controllers computed using various values of $Q_{11}$ and $Q_{22}$ were conducted and the cost of a flight was defined as

$$
c=\left\{\begin{array}{cc}
\bar{\gamma}_{i} & \text { if } \mathbf{x}_{\min } \leq \mathbf{x} \leq \mathbf{x}_{\max } \\
999 & \text { otherwise }
\end{array}\right.
$$

The state limits $\mathbf{x}_{\min }$ and $\mathbf{x}_{\max }$ ensure feasibility of the trajectory and are defined in Table 3 in the Appendix. Here $\bar{\gamma}_{i}$ is the arctangent of the mean flight path angle with respect to the inertial (i.e. ground) reference frame:

$$
\bar{\gamma}_{i}=\frac{\overline{\dot{z}}_{i}}{\overline{\bar{x}}_{i}}
$$

In still air $\overline{\gamma_{i}}$ will be positive, representing a steady glide with $\frac{1}{\bar{\gamma}_{i}}=\frac{L}{D}$. Any value of $\overline{\gamma_{i}}$ less than this steady glide value represents a reduction in energy loss, and negative values of $\bar{\gamma}_{i}$ denote net energy gain. The value $\frac{1}{\bar{\gamma}_{i}}$ can thus be seen as an "effective" L/D.

Figure 3 show surface plots of $\frac{1}{\bar{\gamma}_{i}}$. Note that for the gust field used here $\overline{\gamma_{i}}$ was always positive.

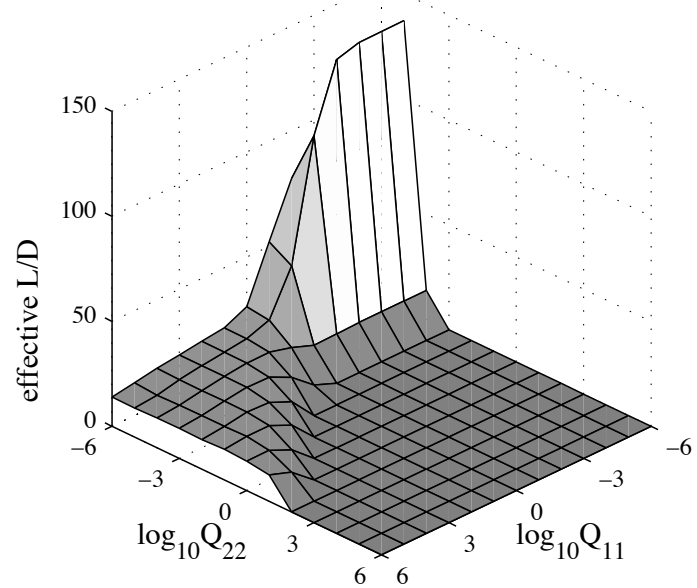

(a)

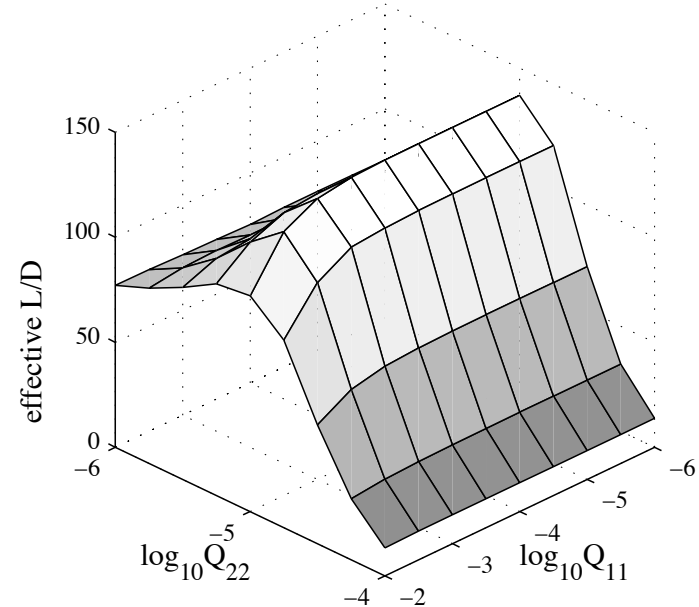

(b)

Figure 3. Surface plots of effective L/D vs. weights. The right hand plot covers a smaller range of $Q_{11}$ and $Q_{22}$.

The peak at very small values of $Q_{11}$ and $Q_{22}$ shows that the critical state to be controlled is $\alpha$. It is interesting to note that while airspeed (weighted by $Q_{22}$ ) is not weighted highly, its importance is not 
zero: the effective L/D peaks when $Q_{22}=1 \times 10^{-5}$. Additionally, it is not critical to track pitch angle $\theta$ : effective L/D remains high even for very small values of $Q_{11}$. The flat region with zero effective L/D denote controllers where state constraints were violated at some point during the simulation.

Note that in steady state gliding flight angle of attack and airspeed are directly related. It should therefore come as no surprise that airspeed is given some weight in the control design. However, tracking an airspeed command in gusty conditions using only elevator is more difficult than tracking an angle of attack command, since the time constant of airspeed with respect to elevator is much slower than the time constant of angle of attack.

Using $Q_{11}=1 \times 10^{-6}$ and $Q_{22}=1 \times 10^{-5}$, the final controller gains are

$$
\mathbf{K}=\left[\begin{array}{llll}
0.3286 & -0.0087 & 1.0773 & 0.2781
\end{array}\right]
$$

The combination of the optimal steady state energy extraction computation of Subsection III.B with the state feedback controller defined above (referred to as "gust soaring controller") enables energy extraction from flight through gusts.

\section{B. Comparison with output-regulating control}

Rather than minimize the error in state regulating, one can minimize the error in output regulating. In an $\mathrm{LQR}$ formulation the cost function becomes

$$
J_{c}=\int\left(\left(\mathbf{y}-\mathbf{y}_{\text {des }}\right)^{T} \mathbf{Q}\left(\mathbf{y}-\mathbf{y}_{\text {des }}\right)+\mathbf{u}^{T} \mathbf{R u}\right) d t
$$

where $\mathbf{y}$ is the vector of outputs and $\mathbf{y}_{\text {des }}$ is the desired output vector.

As with the state regulating case, the problem is choice of weights $\mathbf{Q}$ and $\mathbf{R}$ to achieve desired system performance. Rather than design output feedback controllers, here we show the importance of controlling airspeed in addition to angle of attack by comparing an output regulating controller using angle of attack with the controller of Equation 40. With $Q=1$ and $R=0.1$ (the same values used for these parameters in the state regulating controller designed above), control gains are

$$
\mathbf{K}=\left[\begin{array}{llll}
0.0645 & -0.0002 & 1.2303 & 0.2636
\end{array}\right]
$$

Note that this is still a state feedback controller, but the controller gains differ significantly from those of Equation 40. This reflects the reduced importance placed on regulating pitch angle and airspeed.

A comparison of flight through a sinusoidal gust field using the two controller designs is given in Figure 4. The better performance of the state regulating controller (by a factor of two) shows the importance of some airspeed control: this performance difference is also reflected in Figure 3.

Note the transients which occur over the first few gust wavelengths before steady sinusoidally varying flight develops. This is due in part to the non-smooth gust initiation (the gradient of $\sin x$ is non-zero at $x=0$ ), but may also be due in part to the steady state assumption made when computing the optimal state for energy extraction. 

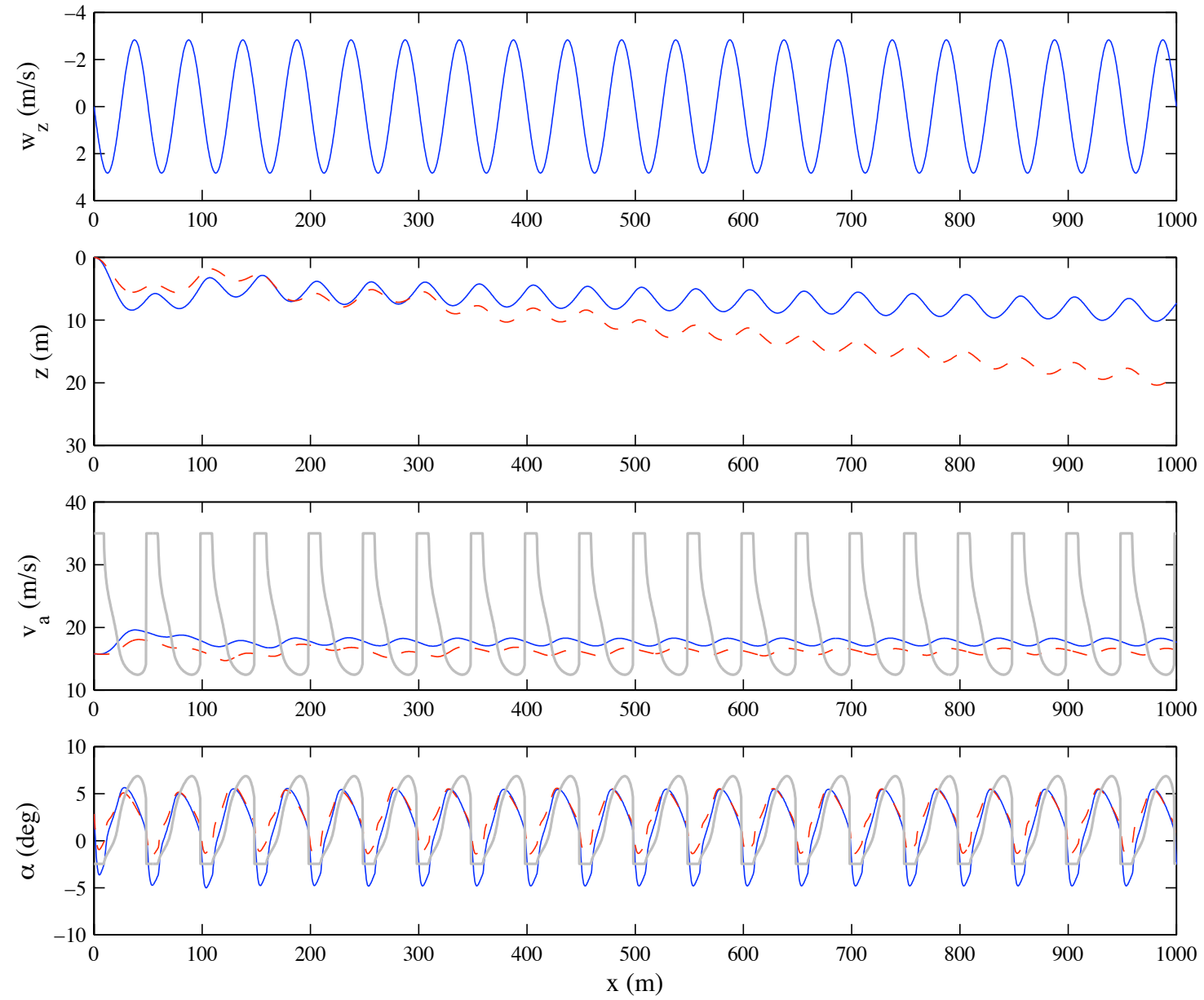

Figure 4. Flight performance comparison of state regulating (solid blue) vs. output regulating (dashed red). For both cases the states to be flown are computed using the steady state energy extraction computation. The upper plot shows vertical wind speed, the second plot show flight path, the third plot shows airspeed, the fourth plot shows angle of attack. The grey line in the second and third plots shows the optimal state for energy maximization computed using the steady state assumption. 


\section{Simulation and Discussion}

Simulations of Flights using the gust soaring controller were conducted for sinusoidally varying gust Sfields and for flight through a field of randomly distributed thermals.

\section{A. Sinusoidal Gust Fields}

To assess the effectiveness of the gust soaring controller over various gust fields and to assess range of gust conditions over which energy extraction is possible a sequence of simulations of flight through sinusoidal gust fields of varying wavelength and root mean square vertical wind velocity were conducted. In all cases flights of $5000 \mathrm{~m}$ were conducted. Results are given in Figure 5.

The closed loop controller was designed for the case of $L_{w}=50 \mathrm{~m}$, so it is to be expected that performance is maximized here. Net energy gain occurs when $\sigma_{z} \gtrsim 2.1 \mathrm{~m} \mathrm{~s}^{-1}$ (the effective flight path angle is greater than zero).

Results presented in Lissaman and Patel ${ }^{6}$ indicate that energy neutral trajectories at this wavelength are achievable with a root mean square gust intensity of approximately $1.13 \mathrm{~m} \mathrm{~s}^{-1}$. However, this assumes: (1) full knowledge of future wind speeds, so that the optimal trajectory can be computed; (2) the optimal trajectory so computed can be flown exactly. Here a sub-optimal state is computed without knowledge of future wind speeds, and elevator control is used to regulate vehicle state to the desired values.

For the shorter wavelength gust field $\left(L_{w}=\right.$ $25 \mathrm{~m}$ ) performance is similar at low gust intensities but becomes degraded at higher gust intensities. It appears that the wind gradients are too steep at this wavelength, and the closed loop controller is unable to maintain adequate regulation.

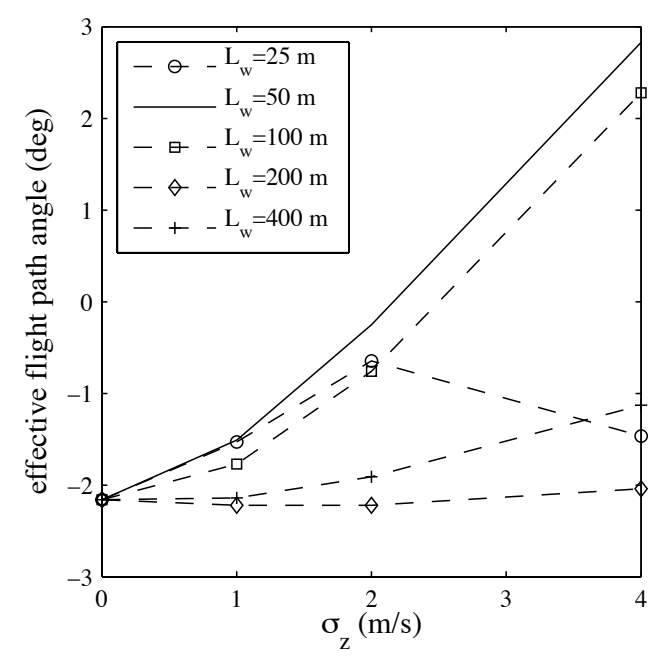

Figure 5. Effective flight path angle for various sinusoidal gust fields. Note that negative flight path angle denotes a downward glide. Best $\mathrm{L} / \mathrm{D}$ for the SB-XC in still air is approximately $27\left(-2.12^{\circ}\right)$.

At $\left(L_{w}=100 \mathrm{~m}\right)$ performance is good over the full range of intensities examined. The reduced performance may be due to a non-optimal closed loop controller, but is more likely due to the smaller wind gradients, reducing the energy that can be obtained.

At $L_{w}=400 \mathrm{~m}$ essentially pure dolphin soaring can occur (since vehicle dynamics are fast compared with gust period). Wind gradients are small, however, and the poor performance of the vehicle at high speeds greatly reduces overall performance compared with the shorter wavelength cases.

The overall poor performance at $\left(L_{w}=200 \mathrm{~m}\right)$ requires further investigation.

\section{B. Thermal Fields}

Since it is not clear that they are appropriate for small and micro UAVs, rather than test the controller on a Dryden or von Karman gust field, here we will simulate flight of the vehicle through a field of randomly placed thermals.

For a single thermal, the vertical wind velocity is computed using a model presented in Gedeon ${ }^{14}$ and used in several studies of optimal flight trajectories: ${ }^{21}$

$$
w_{z}(x)=w_{0} e^{-\left(\frac{x-x_{0}}{R}\right)^{2}}\left[1-\left(\frac{x-x_{0}}{R}\right)^{2}\right]
$$

where $w_{0}$ is the maximum vertical wind speed, $x_{0}$ is the thermal center and $R$ is the thermal radius. This model provides a region of sinking air immediately around the rising central core, which has been oberved by glider pilots. The "area of effect" of a thermal is approximately three times the thermal radius. This thermal profile is shown in Figure 6. 
Table 1. Parameters for thermal field.

\begin{tabular}{ccc}
\hline \hline variable & range & description \\
\hline$w_{0}$ & {$\left[\begin{array}{cc}1 & -5\end{array}\right]$} & maximum vertical wind speed (positive down) \\
$R$ & {$\left[\begin{array}{ll}10 & 100]\end{array}\right.$} & thermal radius \\
$x_{i}-x_{j}$ & {$\left[\left(R_{i}+R_{j}\right) 3\left(R_{i}+R_{j}\right)\right]$} & inter-thermal spacing \\
\hline \hline
\end{tabular}

For a sequence of $N$ thermals the total vertical wind speed is

$$
w_{z}(x)=\sum_{n=1}^{N} w_{0, n} e^{-\left(\frac{x-x_{n}}{R_{n}}\right)^{2}}\left[1-\left(\frac{x-x_{n}}{R_{n}}\right)^{2}\right]
$$

A field of 30 thermals was randomly generated using uniform distributions over the ranges of variables given in Table 1. For the results presented here, the root mean square vertical gust velocity was $1.27 \mathrm{~m} \mathrm{~s}^{-1}$ and the mean vertical gust velocity was $-0.45 \mathrm{~m} \mathrm{~s}^{-1}$ (positive down).

The vehicle was flown for $5000 \mathrm{~m}$ through this thermal field under two conditions: the gust soaring controller and an output regulating controller which

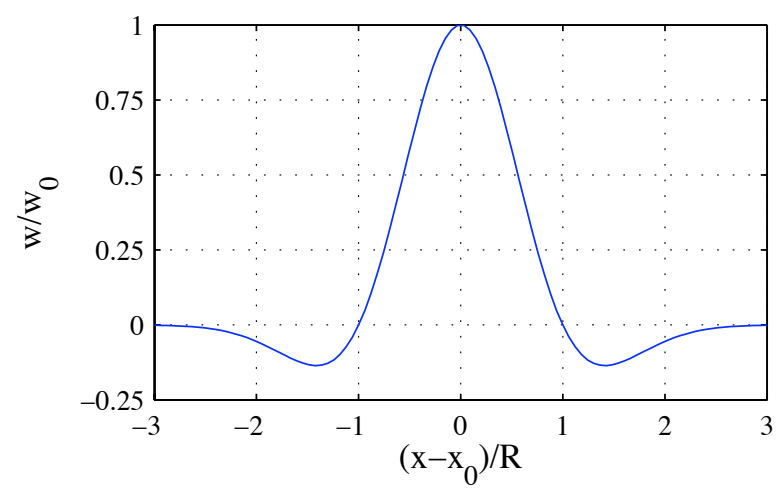

Figure 6. Normalized vertical wind profile for Gedeon thermal model. was set to regulate airspeed to best L/D in still air (15.8 $\mathrm{m} \mathrm{s}^{-1}$ for this aircraft). Results are presented in Figure 7.

The constant speed controller (shown using the dashed red line in Figure 7) gave an effective flight path angle (computed using Equation 39) of $0.65^{\circ}$ (i.e. effective $\mathrm{L} / \mathrm{D}=88$ ). The same thermal field flown using the gust soaring controller gave an effective flight path angle of $0.434^{\circ}$ (i.e. effective $\mathrm{L} / \mathrm{D}=132$ ).

To separate the effect of the mean vertical wind from the gusts, simulation of flight through a constant vertical wind field of $-0.45 \mathrm{~m} \mathrm{~s}^{-1}$ (equal to the mean vertical wind speed of the thermal field) was conducted. For the SB-XC the speed for best L/D in this wind field is $14.7 \mathrm{~m} \mathrm{~s}^{-1}$, and at this speed the effective flight path angle is $0.59^{\circ}$ (i.e. effective $\mathrm{L} / \mathrm{D}=96$ ). A comparison of flight in this constant wind field with the thermal field is shown in Figure 8.

\section{Conclusion}

This paper has presented a closed loop architecture for gust energy extraction for small- and microUninhabited Aerial Vehicles. An equation for rate of change of specific energy for flight through a spatially varying wind field is derived using a full model of aircraft longitudinal dynamics (i.e. vehicle inputs are thrust coefficient, elevator deflection and flap deflection).

To enable closed loop control the state which maximizes instantaneous energy gain using only current measurements of wind speed and gradient is computed. A state feedback controller regulates vehicle state to this optimal energy gain state. A critical problem is designing the feedback control law which maximizes energy gain within this gust soaring architecture.

To avoid unreasonable control inputs the computation of instantaneous energy gain requires and assumption of steady gliding flight. The sequence of states computed using this approach will necessarily result in sub-optimal energy gain, however it significantly reduces computational overhead in addition to obviating the need for full a priori knowledge of the gust field.

A gust soaring controller was designed for a small autonomous glider with only elevator input. The state feedback controller was designed using LQR synthesis, and a search method was used to determine the state weight matrix which maximized energy gain for flight through a sinusoidal gust field. This optimal state weight matrix was used to generate the feedback control law.

Simulations of flights through sinusoidal gust fields of varying wavelength and root mean square verti- 

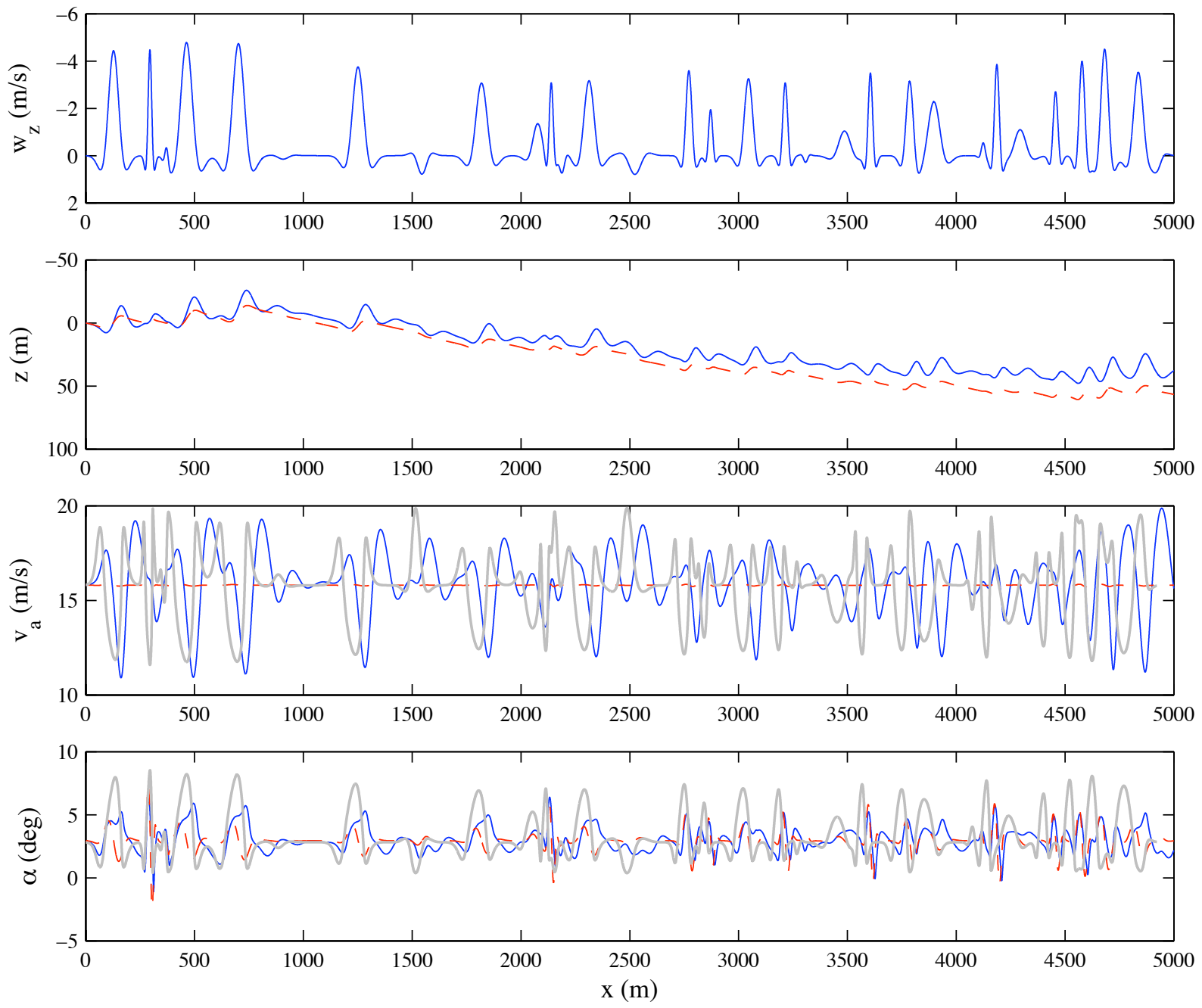

Figure 7. Flight through a field of randomly placed thermals. The solid blue line shows results using the gust soaring controller, the dashed red line shows results from the constant airspeed controller. The upper plot shows the vertical wind speed; the second plot shows the flight path; the third plot shows airspeed flown; the fourth plot shows angle of attack flown. The gray line shows the optimal states for energy maximization computed using the steady state assumption. 

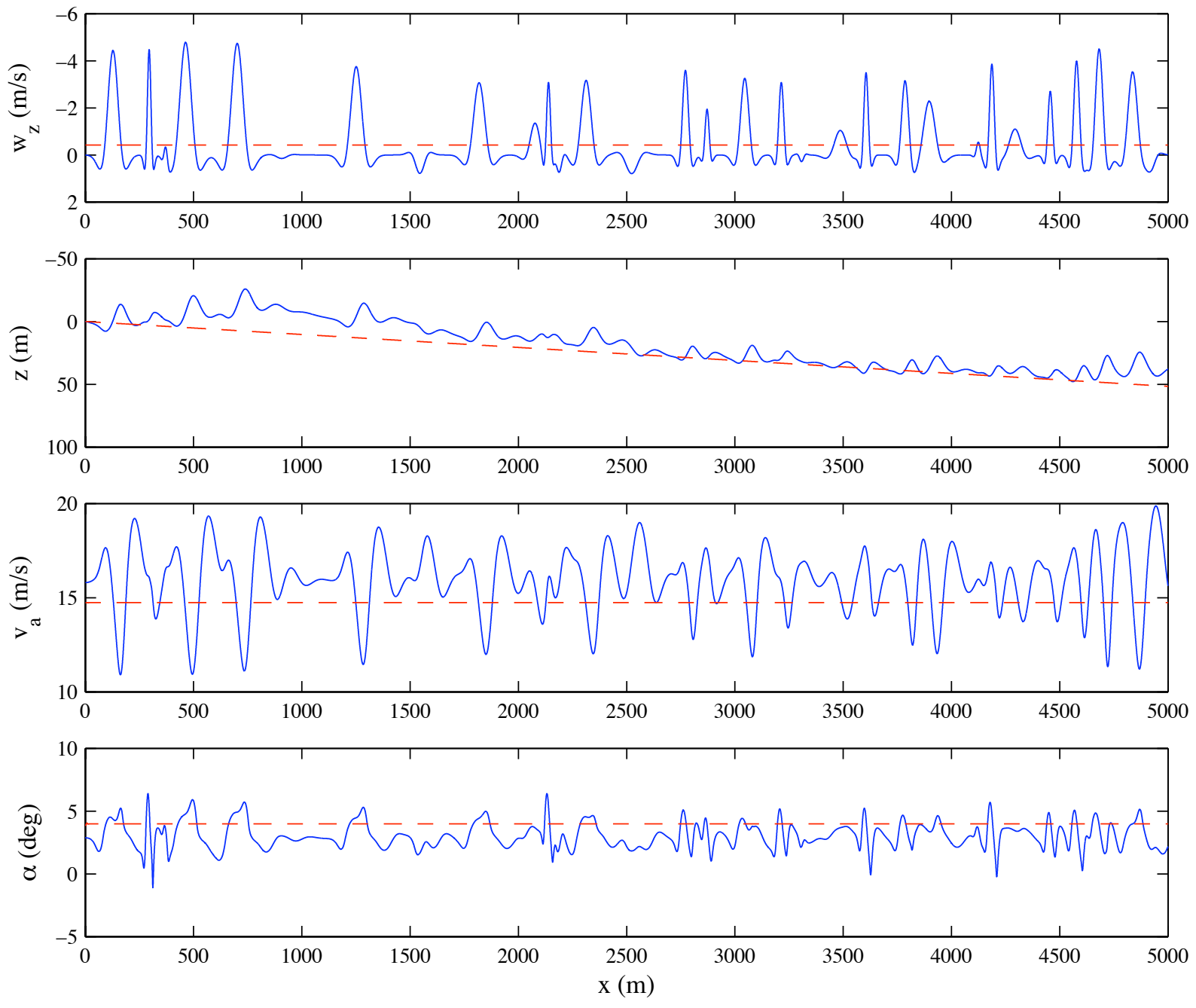

Figure 8. Comparison of flight through a field of randomly placed thermals vs. flight through constant vertical wind field. The solid blue line shows results using the gust soaring controller in the thermal field, the dashed red line shows results from the constant airspeed controller flying in the constant vertical wind field. The upper plot shows the vertical wind speed; the second plot shows the flight path; the third plot shows airspeed flown; the fourth plot shows angle of attack flown. 
cal wind velocity show the effectiveness of this gust soaring controller, and simulation of flight through a thermal field shows that significant improvement in flight performance is possible. Future work will include improvements to the energy maximization computation.

\section{Acknowledgments}

The authors thank Mark Maughmer for many insightful discussions.

\section{References}

${ }^{1}$ Boslough, M. B. E., "Autonomous Dynamic Soaring Platform for Distributed Mobile Sensor Arrays," Tech. Rep. SAND2002-1896, Sandia National Laboratories, Sandia National Laboratories, 2002.

${ }^{2}$ Kiceniuk, T., "Calculations on Soaring in Sink," Technical Soaring, Vol. 25, No. 4, October 2001, pp. 228-230.

${ }^{3}$ Allen, M. J. and Lin, V., "Guidance and Control of an Autonomous Soaring Vehicle with Flight Test Results," AIAA Aerospace Sciences Meeting and Exhibit, AIAA Paper 2007-867, American Institute of Aeronautics and Astronautics, Reno, Nevada, January 2007.

${ }^{4}$ Phillips, W. H., "Propulsive Effects due to Flight through Turbulence," Journal of Aircraft, Vol. 12, No. 7, July 1975, pp. 624-626.

${ }^{5}$ Patel, C. K. and Kroo, I., "Control Law Design for Improving UAV Performance using Wind Turbulence," $A I A A$ Aerospace Sciences Meeting and Exhibit, AIAA Paper 2006-0231, American Institute of Aeronautics and Astronautics, Reno, Nevada, January 2006.

${ }^{6}$ Lissaman, P. B. S. and Patel, C. K., "Neutral Energy Cycles for a Vehicle in Sinusoidal and Turbulent Vertical Gusts," 45th AIAA Aerospace Sciences Meeting and Exhibit, AIAA Paper 2007-863, American Institute of Aeronautics and Astronautics, Reno, Nevada, January 2007.

${ }^{7}$ MacCready Jr., P. B., "Optimum Airspeed Selector," Soaring, January-February 1958, pp. 10-11.

${ }^{8}$ Arho, R., "Distance Estimation Error and Stationary Optimal Gliding," OSTIV Publication XII, Organisation Scientifique et Technique Internationale du Vol á Voile, 1972.

${ }^{9}$ Metzger, D. E. and Hedrick, J. K., "Optimal Flight Paths for Soaring Flight," Journal of Aircraft, Vol. 12, No. 11, 1975, pp. $867-871$.

${ }^{10}$ de Jong, J. L., "The Convex Combination Approach: A Geometric Approach to the Optimization of Sailplane Trajectories," OSTIV Publication XVI, Organisation Scientifique et Technique Internationale du Vol à Voile, 1981, pp. 182-201.

${ }^{11}$ Cochrane, J. H., "MacCready Theory with Uncertain Lift and Limited Altitude," Technical Soaring, Vol. 23, No. 3, July 1999, pp. 88-96.

${ }^{12}$ Irving, F., "The Energy Loss in Pitching Manoeuvres," Proceedings of the XVI OSTIV Congress, Organisation Scientifique et Technique Internationale du Vol à Voile, 1978.

${ }^{13}$ Gedeon, J., "The Influence of Sailplane Performance and Thermal Strength on Optimal Dolphin-Flight Transition Piloting Techniques," Proceedings of the XV OSTIV Congress, Organisation Scientifique et Technique Internationale du Vol à Voile, 1976.

${ }^{14}$ Gedeon, J., "Dynamic Analysis of Dolphin Style Thermal Cross Country Flight," Proceedings of the XIV OSTIV Congress, Organisation Scientifique et Technique Internationale du Vol à Voile, 1974

${ }^{15}$ Zhao, Y. J., "Optimal Patterns of Glider Dynamic Soaring," Optimal Control Applications and Methods, Vol. 25, 2004, pp. 67-89.

${ }^{16}$ Zhao, Y. J. and Qi, Y. C., "Minimum Fuel Powered Dynamic Soaring of Unmanned Aerial Vehicles Utilizing Wind Gradients," Optimal Control Applications and Methods, Vol. 25, 2004, pp. 211-233.

${ }^{17}$ Sachs, G. and Mayrhofer, M., "Shear Wind Strength Required for Dynamic Soaring at Ridges," Technical Soaring, Vol. 25, No. 4, October 2001, pp. 209-215.

${ }^{18}$ Sachs, G., "Minimum Shear Wind Strength Required for Dynamic Soaring of Albatrosses," Ibis, Vol. 147, 2005 , pp. 1-10.

${ }^{19}$ Pennycuick, C. J., "Gust Soaring as a Basis for the Flight of Petrels and Albatrosses (Procellariiformes)," Avian Science, Vol. 2, No. 1, 2002, pp. 1-12.

${ }^{20}$ Lissaman, P., "Wind Energy Extraction by Birds and Flight Vehicles," 43rd AIAA Aerospace Sciences Meeting and Exhibit, AIAA Paper 2005-241, American Institute of Aeronautics and Astronautics, Reno, Nevada, January 2005.

${ }^{21}$ Qi, Y. C. and Zhao, Y. J., "Energy-Efficient Trajectories of Unmanned Aerial Vehicles Flying Through Thermals," Journal of Aerospace Engineering, Vol. 18, No. 2, April 2005, pp. 84-92. 


\section{Appendix: Vehicle Properties}

Simulation results are based on the RnR products SB-XC radio control glider. Parameters in Table 2 were obtained from a drag buildup computation, state limits in Table 3 were defined to limit states to "reasonable" bounds.

Note that a fourth order polynomial is used to relate $C_{D}$ to $C_{L}$ : this provided a better fit to the computed data over the full speed range.

Table 2. Parameters for SB-XC glider.

\begin{tabular}{ccc}
\hline \hline variable & value & description \\
\hline $\mathrm{m}$ & $10 \mathrm{~kg}$ & mass \\
$\mathrm{b}$ & $4.34 \mathrm{~m}$ & span \\
$\mathrm{c}$ & $0.232 \mathrm{~m}$ & MAC \\
$\mathrm{S}$ & $1 \mathrm{~m}^{2}$ & wing area \\
$I_{y y}$ & $1.87 \mathrm{~kg} \cdot \mathrm{m}^{2}$ & \\
$C_{L 0}$ & 0.37 & \\
$C_{L_{\alpha}}$ & $5.54 / \mathrm{rad}$ & \\
$C_{L_{Q}}$ & $-3.255 \mathrm{~s} / \mathrm{rad}$ & \\
$C_{L_{\dot{\alpha}}}$ & $-0.651 \mathrm{~s} / \mathrm{rad}$ & \\
$C_{L_{\delta_{e}}}$ & $-0.37 / \mathrm{rad}$ & \\
$C_{L_{\delta_{f}}}$ & $1.63 / \mathrm{rad}$ & \\
$f_{L D}(\varphi)$ & $0.1723 \varphi^{4}-0.3161 \varphi^{3}+0.2397 \varphi^{2}$ & \\
& $-0.0624 \varphi+0.0194$ & \\
$C_{D_{\delta_{e}}}$ & $0 / \mathrm{rad}$ & \\
$C_{D_{\delta_{f}}}$ & $0.042 / \mathrm{rad}$ & \\
$C_{m 0}$ & 0 & \\
$C_{m_{\alpha}}$ & $-1.02 / \mathrm{rad}$ & \\
$C_{m_{Q}}$ & $-14.6 \mathrm{~s} / \mathrm{rad}$ & \\
$C_{m_{\delta_{e}}}$ & $1.6275 / \mathrm{rad}$ & \\
$C_{m_{\delta_{f}}}$ & $-0.254 / \mathrm{rad}$ & \\
\hline \hline
\end{tabular}

Table 3. State limits and control saturation for SB-XC glider.

\begin{tabular}{|c|c|c|}
\hline state/control & range & description \\
\hline$\theta$ & {$\left[\begin{array}{ll}-45^{\circ} 45^{\circ}\end{array}\right]$} & pitch \\
\hline$v_{a}$ & {$[11 \mathrm{~m} / \mathrm{s} 35 \mathrm{~m} / \mathrm{s}]$} & airspeed \\
\hline$\alpha$ & {$\left[\begin{array}{ll}-2^{\circ} & 12^{\circ}\end{array}\right]$} & angle of attack \\
\hline$Q$ & {$\left[\begin{array}{lll}-999 & 999\end{array}\right]$} & pitch rate \\
\hline$\delta_{e}$ & {$\left[\begin{array}{ll}-20^{\circ} & 20^{\circ}\end{array}\right]$} & elevator deflection \\
\hline
\end{tabular}

